\title{
Free energies of crystals computed using Einstein crystal with fixed center of mass and different spring constants
}

\author{
Vikram Khanna, ${ }^{1}$ Jamshed Anwar, ${ }^{2}$ Daan Frenkel, ${ }^{3}$ Michael F. Doherty, ${ }^{1}$ and Baron Peters ${ }^{4,5}$, a) \\ 1) Department of Chemical Engineering, University of California Santa Barbara, Santa Barbara, California 93106 , \\ USA \\ 2) Department of Chemistry, Lancaster University, Lancaster LA1 4YW, United Kingdom \\ ${ }^{3)}$ Department of Chemistry, University of Cambridge, Cambridgeshire CB2 1EW, \\ United Kingdom \\ ${ }^{4)}$ Department of Chemical and Biomolecular Engineering, University of Illinois at Urbana-Champaign, Urbana, \\ Illinois 61801, USA \\ ${ }^{5)}$ Department of Chemistry, University of Illinois at Urbana-Champaign, Urbana, Illinois 61801, \\ $U S A$
}

(Dated: 9 March 2021)

Free energies of crystals computed using a center of mass constraint require a finite-size correction, as shown in previous work by Polson et al. Their reference system is an Einstein crystal with equal spring constants. In this paper we extend the work of Polson et al. to the case of different spring constants. The generalization is convenient for constraining the center of mass in crystals with atoms of different masses and it helps to optimize the free energy calculations. To test the theory we compare the free energies of LiI and $\mathrm{NaCl}$ crystals from calculations with different spring constants to those computed using equal spring constants. Using these center of mass finite size corrections, we compute the true free energies of these crystals for different system sizes to eliminate the intrinsic finite-size effects. These calculations help demonstrate the size of these finite-size corrections relative to other contributions to the absolute free energy of the crystals.

\section{INTRODUCTION}

Solid phase free energy computations ${ }^{1-6}$ are widely used to predict fluid-solid equilibria, ${ }^{7-11}$ solid-solid equilibria $^{12}$ and relative stability of polymorphs. ${ }^{13,14}$ The Frenkel-Ladd method ${ }^{3}$ computes the free energy difference between the solid under consideration and an Einstein crystal, a reference system whose free energy is analytically known. The method uses thermodynamic integration to transform between the Einstein crystal and real solid. To supress a weak divergence of the integrand used in computing the free energy, it is recommended to fix the center of mass (COM) of the system. ${ }^{3,8,15,16}$ To compute the absolute free energy of the unconstrained crystal we need to correct for the effect of imposing this constraint in the calculations. The numerical value of the free energy correction per atom (or molecule) tends to zero as the system size tends to infinity, hence it is a finite-size correction. Polson et al. ${ }^{15}$ derived an $\mathcal{O}(\ln N / N)$ term in transformation from constrained to unconstrained crystals as a finite-size correction. Their calculation is based on an Einstein crystal with fixed center of mass and equal spring constants. Note that an alternate method, the Einstein molecule method, ${ }^{4,6,17,18}$ does not require a center of mass constraint or the associated corrections. However, this paper is aimed at completing the theory for the Einstein crystal method.

For crystals comprised of atoms with different atomic masses, different spring constants (in the Einstein crys-

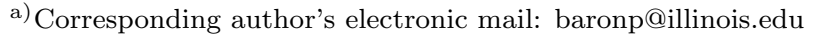

tal approach ${ }^{3}$ ) can help to maintain the center of mass ${ }^{8}$ constraint (if the molecular dynamics package does not have built-in features to achieve the same) by choosing mass-scaled spring constants that equate the angular frequencies. See supplementary material for details. Alternatively, different spring constants can help to optimize the numerical integration to compute the free energy difference by choosing spring constants that reproduce the mean-squared displacement (MSD) of atoms in the real crystal $^{16}$ (referred to as MSD-based springs in this article).

In this paper, we extend the finite-size corrections of Polson et al. ${ }^{15}$ to the case of different spring constants in an Einstein crystal. To test the results we compare the solid free energies of $\mathrm{LiI}$ and $\mathrm{NaCl}$ crystals from three calculations: i) mass-scaled springs that exert a null force on the system and help constrain the COM without the need of built-in functions to constrain the COM, ii) MSD-based springs that reproduce the meansquared displacement (MSD) of atoms in the real crystal that help optimize the numerical integration, and iii) calculations with a set of equal springs. A fourth option, not explored here, is to artificially set all masses and spring constants to equal values, respectively, and then analytically recover the free energies for the real masses, e.g. using equations in Polson et al. ${ }^{15}$ For the MSD-based and equal spring sets we make use of the built-in center of mass constraint features in the molecular dynamics code $\mathrm{LAMMPS}^{19}$. These calculations confirm that the two procedures (employing different and equal springs) are equivalent. They also demonstrate the accuracy gained by the use of spring constants that reproduce the MSD of atoms in the real crystal along with the relative magni- 
tudes of the finite-size corrections and other contributions to the absolute free energy of the crystal.

Note that in addition to the finite size correction due to the COM constraint in the calculations, the free energy of solids presents an intrinsic system size dependence as shown by Vega et al. ${ }^{4}$ These finite size corrections need to be computed by repeating the free energy calculations for several system sizes and extrapolating to infinite size. This intrinsic finite size effect is also computed for the LiI and $\mathrm{NaCl}$ crystal systems to emphasize the difference between the two different types of finite size effects entering the solid free energy calculations., i.e., the one stemming due to the COM constraint in the Einstein crystal method and the other, the intrinsic finite size effect related to the cutoff in the phonon spectrum introduced by the finite lattice size. ${ }^{20}$

\section{THEORY}

The free energy $(F)$ of a real crystal $(\mathrm{C})$ is computed using the following path:

$$
F_{E C} \rightarrow F_{E C}^{C M} \rightarrow F_{C}^{C M} \rightarrow F_{C}
$$

where CM indicates a center of mass constraint and EC refers to an Einstein crystal.

Therefore,

$$
F_{C}=F_{E C}+\left(F_{E C}^{C M}-F_{E C}\right)+\left(F_{C}^{C M}-F_{E C}^{C M}\right)+\left(F_{C}-F_{C}^{C M}\right)
$$

where the absolute free energy of the Einstein crystal can be obtained from its analytically computable partition function:

$$
\beta F_{E C}=-\ln \left(Q_{E C}\right)
$$

and the free energy differences are

$$
\begin{gathered}
\beta\left(F_{E C}^{C M}-F_{E C}\right)=-\ln \left(\frac{Q_{E C}^{C M}}{Q_{E C}}\right) \\
\beta\left(F_{C}^{C M}-F_{E C}^{C M}\right)=\beta \int_{\lambda=0}^{\lambda=1}\left\langle\frac{d U}{d \lambda}\right\rangle_{\lambda}^{C M} d \lambda \\
\beta\left(F_{C}-F_{C}^{C M}\right)=-\ln \left(\frac{Q_{C}}{Q_{C}^{C M}}\right)
\end{gathered}
$$

In each of these formulas, $Q$ is a partition function, $\beta=$ $\left(k_{B} T\right)^{-1}, \lambda$ is a coupling parameter, $U$ is a $\lambda$-dependent potential energy function that interpolates between that of the EC and $\mathrm{C}$ systems.

\section{A. Spring Constants and Finite Size Corrections for Einstein Crystals}

When all atoms have a common spring constant $(k)$, the Helmholtz free energy of a crystal with $N_{m o l}$ molecules composed of a total of $N$ atoms, is given by Polson et al. ${ }^{15}$ as:

$$
\begin{aligned}
\beta F_{C} & =\sum_{i=1}^{N} \ln \left(\frac{\beta k \Lambda_{i}^{2}}{2 \pi}\right)^{3 / 2} \\
& +\beta \int_{\lambda=0}^{\lambda=1}\left\langle\frac{d U}{d \lambda}\right\rangle_{\lambda}^{C M} d \lambda \\
& -\ln \left[\left(\frac{\beta k}{2 \pi \sum_{i=1}^{N} \mu_{i}^{2}}\right)^{3 / 2}\left(\frac{V}{N_{m o l}}\right)\right]
\end{aligned}
$$

where $\Lambda_{i}=\left(\beta h^{2} /\left(2 \pi m_{i}\right)\right)^{1 / 2}, h$ is Planck's constant, $m_{i}$ is the mass of atom $i, V$ is the volume of the system, and $\mu_{i}=m_{i} / \sum_{i=1}^{N} m_{i}$.

We have combined terms in the result as given in Polson et al. so that all arguments of logarithms are dimensionless.

Note that the corresponding equations in ref. [8], for atoms with different spring constants cannot be combined to give a dimensionless argument to the logarithm. The error introduced in that study, however, was marginal as the spring constants were similar. Using our revised result shown in equation (10), the free energy in Ref. [8] is revised to $-97.44 \pm 0.02 N_{\text {mol }} k_{B} T$ from -97.75 $\pm 0.02 N_{m o l} k_{B} T$. Since the estimated errors in the free energy of the fluid phase were larger $\left(\geq 1 N_{\text {mol }} k_{B} T\right)$ than the introduced error in the Einstein crystal calculation, the melting point prediction of the study is unaffected, and indeed has been confirmed by independent density of states calculations. ${ }^{21}$ In this work we provide a revised version of equation (4) for systems where atoms have different spring constants.

Since the part of the finite-size correction involving spring constants emerges from equation (3a), (see Appendix A), we derive the configurational partition function ratio, $Z_{E C}^{C M} / Z_{E C}$ here for an $\mathrm{EC}$ with different spring constants $\left(k_{i}, i=1,2, \ldots, N\right)$.

For 1-Dimension (x-direction), the configurational partition function of an Einstein crystal with a COM constraint is:

$$
Z_{E C, x}^{C M}=\int d x^{N} \prod_{i=1}^{N} \exp \left[-\frac{\beta k_{i}}{2} x_{i}^{2}\right] \delta\left(\sum_{i=1}^{N} \mu_{i} x_{i}\right)
$$

Without loss of generality, we are letting all particles be attached by a spring to a point at the origin. This makes $x_{i}$ the displacement of atom $i$ in the x-direction from the tether point (origin here).

We make the following variable transformation:

$$
\xi_{i}=k_{i}^{1 / 2} x_{i}
$$

with Jacobian determinant

$$
J=\left|\prod_{i=1}^{N} k_{i}^{-1 / 2}\right|
$$


Therefore,

$$
\begin{aligned}
Z_{E C, x}^{C M} & =\int d \xi^{N} J \prod_{i=1}^{N} \exp \left[-\frac{\beta}{2} \xi_{i}^{2}\right] \delta\left(\sum_{i=1}^{N} \frac{\mu_{i}}{k_{i}^{1 / 2}} \xi_{i}\right) \\
& =J\left(\frac{\beta}{2 \pi \sum_{i=1}^{N} \frac{\mu_{i}^{2}}{k_{i}}}\right)^{1 / 2} \prod_{i=1}^{N}\left(\frac{2 \pi}{\beta}\right)^{1 / 2} \\
& =\left(\frac{\beta}{2 \pi \sum_{i=1}^{N} \frac{\mu_{i}^{2}}{k_{i}}}\right)^{1 / 2} \prod_{i=1}^{N}\left(\frac{2 \pi}{\beta k_{i}}\right)^{1 / 2} \\
& =\left(\frac{\beta}{2 \pi \sum_{i=1}^{N} \frac{\mu_{i}^{2}}{k_{i}}}\right)^{1 / 2} Z_{E C, x}
\end{aligned}
$$

where $Z_{E C, x}$ is the integral in equation (5), but without the center of mass constraint. See supplementary material for additional details. The extension to three dimensions just results in three factors of $\left(\beta / 2 \pi \sum_{i}\left[\mu_{i}^{2} / k_{i}\right]\right)^{1 / 2}$.

$$
\frac{Z_{E C}^{C M}}{Z_{E C}}=\left(\frac{\beta}{2 \pi \sum_{i=1}^{N} \frac{\mu_{i}^{2}}{k_{i}}}\right)^{3 / 2}
$$

Using equation (9) in place of equation (A.5a) in the derivation shown in Appendix A, we get

$$
\begin{aligned}
\beta F_{C} & =\sum_{i=1}^{N} \ln \left(\frac{\beta k_{i} \Lambda_{i}^{2}}{2 \pi}\right)^{3 / 2} \\
& +\beta \int_{\lambda=0}^{\lambda=1}\left\langle\frac{d U}{d \lambda}\right\rangle_{\lambda}^{C M} d \lambda \\
& -\ln \left[\left(\frac{\beta}{2 \pi \sum_{i=1}^{N} \frac{\mu_{i}^{2}}{k_{i}}}\right)^{3 / 2}\left(\frac{V}{N_{\text {mol }}}\right)\right]
\end{aligned}
$$

for an Einstein crystal system with different spring constants.

Equation (10) gives the free energy of a real crystal computed using an EC system constituting different spring constants. Note that equation (10) is properly dimensionless. Moreover, equation (10) collapses to equation (4) when $k_{i}=k$, i.e., when all spring constants are equal.

\section{B. Constraining the center of mass}

In molecular dynamics (MD) simulations with standard force fields, the center of mass can be fixed by beginning with zero total momentum and not adding external forces. For the Einstein crystal, the springs do exert external forces so additional measures are needed.

A simple way to fix the center of mass in a system with springs begins by making the spring constants proportional to the atom masses, so that all atoms have the same frquency. When all oscillators have frequency $\omega$, the center of mass evolves as

$$
\mathbf{x}_{C M}(t)=\omega^{-1} \mathbf{v}_{C M}^{o} \sin (\omega t)+\mathbf{x}_{C M}^{o} \cos (\omega t)
$$

where, $\mathbf{v}_{C M}^{o}$ is the initial center of mass velocity, and $\mathbf{x}_{C M}^{o}$ is the initial displacemet of the center of mass from the tether points' (lattice positions') center of mass. See supplementary material for details.

Clearly, we can maintain $\mathbf{x}_{C M}=0$ by choosing spring constants proportional to the atom masses, so that all atoms have the same frquency and setting initial conditions such that $\mathbf{v}_{C M}^{o}=\mathbf{x}_{C M}^{o}=0$.

We note that certain MD packages such as LAMMPS do not need to have a net zero external force on the system to constrain the center of mass. This is achieved by thermostating all degrees of freedom except the center of mass and shifting all atom coordinates after every timestep (equal to the drift in center of mass) to recenter the system to the initial value of the center of mass. This recentering does not alter the dynamics of the system or change the relative coordinates of any pair of atoms.

\section{Thermodynamic Integration}

To compute the free energy difference between the crystal of interest and the Einstein crystal, i.e., $F_{C}^{C M}-$ $F_{E C}^{C M}$, we use thermodynamic integration (T.I.) ${ }^{22}$ with a linear homotopy ${ }^{23}$

$$
U\left(T, V_{C} ; \lambda\right)=(1-\lambda) U_{E C}\left(T, V_{C}\right)+\lambda U_{C}\left(T, V_{C}\right)
$$

where $U\left(T, V_{C} ; \lambda\right)$ is the potential energy function and $\lambda$ is the coupling parameter. Also, $U_{E C}$ and $U_{C}$ are the potential energy functions of the Einstein crystal and the crystal of interest (described by the chosen force field), respectively. Therefore

$$
\begin{aligned}
F_{C}^{C M}-F_{E C}^{C M} & =\int_{\lambda=0}^{\lambda=1}\left\langle\frac{d U}{d \lambda}\right\rangle_{\lambda}^{C M} d \lambda \\
& =\int_{\lambda=0}^{\lambda=1}\left\langle U_{C}-U_{E C}\right\rangle_{\lambda}^{C M} d \lambda
\end{aligned}
$$

where, $\langle d U / d \lambda\rangle_{\lambda}^{C M}$ is evaluated by computing an average of $\left(U_{C}-U_{E C}\right)$ over configurations in the $\lambda$ state canonical ensemble. Note, for $0<\lambda<1$, equation (11) is still applicable. See supplementary material for details.

\section{SIMULATION DETAILS}

We model LiI and $\mathrm{NaCl}$ using the Joung Cheatham force field (the version optimized for SPC/E) ${ }^{24}$ All MD simulations were carried out using LAMMPS. ${ }^{19}$

The NVT simulations for computing the free energies were setup using the interionic distances reported by 
Joung et al. ${ }^{24}$, of $3.05 \AA$, and $2.89 \AA$ for LiI and $\mathrm{NaCl}$, respectively. A super cell measuring $36.6 \AA \times 36.6 \AA \times 36.6$ $\AA$ comprising 864 ion pairs was used for LiI, and a super cell measuring $34.68 \AA \times 34.68 \AA \times 34.68 \AA$ comprising 864 ion pairs was used for $\mathrm{NaCl}$. This is equivalent to 6 $\times 6 \times 6$ unit cells for both crystal systems. To compute the intrinsic finite-size effects, systems comprising $7 \times 7$ $\times 7,8 \times 8 \times 8$, and $9 \times 9 \times 9$ unit cells were used for both crystals.

The Lorentz-Berthelot mixing rules were used for computing the interatomic pair coefficients. We used a time step of 1 fs. Nonbonded interactions were cutoff at 1.6 $\mathrm{nm}$, and $1.1 \mathrm{~nm}$ for $\mathrm{LiI}$ and $\mathrm{NaCl}$, respectively, with long range electrostatics handled by LAMMPS' ParticleParticle-Particle-Mesh (PPPM) summation ${ }^{25,26}$ and a switching function applied for Lennard-Jones interactions between 1.4-1.6 nm, and 0.9-1.1 $\mathrm{nm}$ for LiI and $\mathrm{NaCl}$, respectively.

A 25 point Gauss Legendre quadrature method ${ }^{27}$ was used to evaluate the integral in equation 13 . The NVT simulations were run for a total of $8 \mathrm{~ns}$, of which the initial 2 ns were used for equilibration and then discarded. Data every 1 ps was used to compute the thermodynamic averages for $\mathrm{LiI}$ and $\mathrm{NaCl}$ systems (the autocorrelation times for $\langle d U / d \lambda\rangle_{\lambda}$ were approximately in the range of 50 - $400 \mathrm{fs}$, therefore a 1 ps sampling frequency provides independent samples for each $\lambda$ state. See supplementary material for autocorrelation time calculations). The spring constants for the reference Einstein crystals are chosen to reproduce the mean square displacement of lithium and sodium ions at 300K. For the different springs case, respective spring constants are scaled to compute the counter ion's spring constant such that $k_{i} / m_{i}$ is constant. Table I reports the numerical values used in this study.

TABLE I: Masses (a.m.u) \& spring constants $\left(\mathrm{k}_{\mathrm{B}} \mathrm{T} / \AA^{2}\right)$

\begin{tabular}{|c|c|c|c|c|}
\hline & \multicolumn{2}{|c|}{$L i I$} & \multicolumn{2}{|c|}{$\mathrm{NaCl}$} \\
\hline & $\mathbf{L i}^{+}$ & $\mathbf{I}^{-}$ & $\mathrm{Na}^{+}$ & $\mathrm{Cl}^{-}$ \\
\hline mass & 6.941 & 126.904 & 22.990 & 35.450 \\
\hline $\mid k_{i}$ using mass-scaled springs $\mid$ & 35.866 & | 655.749 & $77.543 \mid$ & 119.581 \\
\hline $\mid k_{i}$ using MSD-based springs $\mid$ & $\mid 35.866$ & | 67.121 & $77.543 \mid$ & 85.167 \\
\hline$k_{i}$ using equal springs & $\mid 35.866$ & | 35.866 & $77.543 \mid$ & 77.543 \\
\hline
\end{tabular}

For the MSD-based and equal springs cases, to constrain the center of mass in LAMMPS, we use the temp/com command in conjuction with the langevin thermostat to thermostat all degrees of freedom except the center of mass. We use the recenter command to correct for the drift in the center of mass after every time step.

\section{RESULTS AND DISCUSSION}

For each crystal system (i.e., LiI and $\mathrm{NaCl}$ ) we compute free energies using mass-scaled, MSD-based and equal spring constants. Table II shows the dimensionless Helmholtz free energies per ion pair. All components of equation 10 are reported to show the relative magnitudes of corrections. See supplementary material for plots of the T.I. integrands. The free energies should match for the two procedures, i.e., with different and equivalent springs. As seen in Table II the free energies computed using different spring constants are in excellent agreement with the free energies computed using equivalent spring constants, thereby successfully testing the theory. Also, the use of MSD-based spring constants optimizes the calculations as can be seen in the near $24 \%$ uncertainty reduction for LiI when compared to the equal spring case. This is because the MSD of the Li and I atoms differ significantly as can be seen by the MSD-based spring constants. In the $\mathrm{NaCl}$ calculations, the MSD of the two atoms are similar, leading to marginal accuracy gains on using MSD-based springs. We also compute the free energy of $\mathrm{NaCl}$ crystal at 298 $\mathrm{K}$ to compare with Aragones et al. ${ }^{6}$ Our free energy estimate at $298 \mathrm{~K}$ is $-311.0340 \pm 0.0002 \mathrm{~N}_{\text {mol }} \mathrm{k}_{\mathrm{B}} \mathrm{T}$ (using $\Lambda_{N a}=\Lambda_{C l}=1 \AA$ ), which is in excellent agreement with their result of $-311.10 \pm 0.1 \mathrm{~N}_{\text {mol }} \mathrm{k}_{\mathrm{B}} \mathrm{T}$.

As seen in Figures 1 and 2, the solid free energies of LiI and $\mathrm{NaCl}$ demonstrate a significant system size dependence, i.e. the free energy size dependence is greater than the calculation uncertainty. Tables III and IV show the accounting of the COM constraint finite size correction for each system size for $\mathrm{LiI}$ and $\mathrm{NaCl}$, respectively.

We use a linear model to capture the intrinsic finite size effect and predict free energy in the thermodynamic limit: ${ }^{4}$

$$
\beta f\left(N_{m o l}\right)=\beta f\left(N_{m o l} \rightarrow \infty\right)+\frac{d_{1}}{N_{m o l}}
$$

The value of the slopes $\left(d_{1}\right)$ for $\mathrm{LiI}$ and $\mathrm{NaCl}$ are -13.1 \pm 0.2 , and $-14.7 \pm 0.2$, respectively.

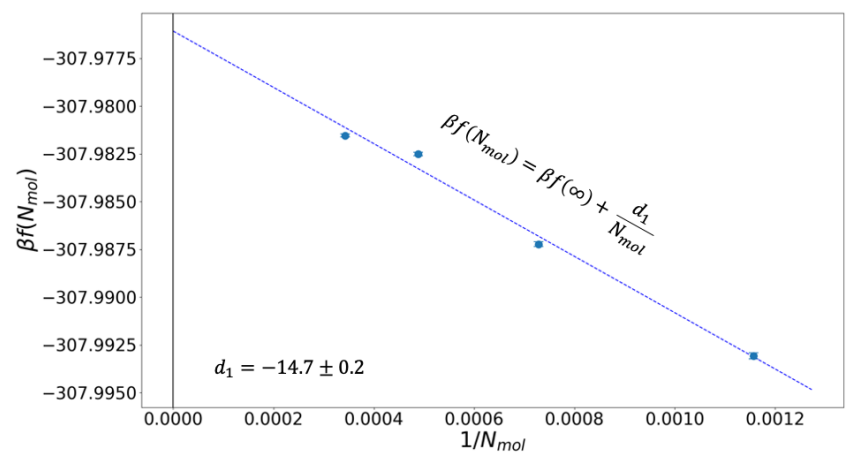

FIG. 1: Intrinsic system-size dependence of LiI crystal's free energy 
TABLE II: Absolute dimensionless Helmholtz free energy $\left(\beta f=\beta F / N_{m o l}\right)$ calculation of LiI and $\mathrm{NaCl}$ (per ion pair) at $300 \mathrm{~K}$. See supplementary material for details of the column headings shown in this table.

\begin{tabular}{|c|c|c|c|c|c|}
\hline \hline Crystal system & $\beta f_{E C}$ & $\beta\left(f_{E C}^{C M}-f_{E C}\right)^{*}$ & $\beta \Delta f_{E C \rightarrow C}^{C M}$ & $\beta\left(f_{C}-f_{C}^{C M}\right)^{*}$ & $\beta f_{C}$ \\
\hline $\begin{array}{c}\text { LiI } \\
\text { mass-scaled springs }\end{array}$ & -0.53908 & -0.01990 & $-307.42991 \pm 0.00028$ & -0.00467 & $-307.99356 \pm 0.00028$ \\
\hline $\begin{array}{c}\text { LiI } \\
\text { MSD-based springs }\end{array}$ & -3.95800 & -0.01603 & $-304.01438 \pm 0.00016$ & -0.00467 & $-307.99308 \pm 0.00016$ \\
\hline $\begin{array}{c}\text { LiI } \\
\text { equiv. springs }\end{array}$ & -4.89807 & -0.01494 & $-303.07519 \pm 0.00021$ & -0.00467 & $-307.99286 \pm 0.00021$ \\
\hline $\begin{array}{c}\text { NaCl } \\
\text { mass-scaled springs }\end{array}$ & -1.81876 & -0.01772 & $-317.07633 \pm 0.00015$ & -0.00449 & $-318.91719 \pm 0.00015$ \\
\hline $\begin{array}{c}\text { NaCl } \\
\text { MSD-based springs }\end{array}$ & -2.32772 & -0.01734 & $-316.56798 \pm 0.00015$ & -0.00449 & $-318.91753 \pm 0.00015$ \\
\hline $\begin{array}{c}\text { NaCl } \\
\text { equiv. springs }\end{array}$ & -2.46850 & -0.01723 & $-316.42744 \pm 0.00016$ & -0.00449 & $-318.91755 \pm 0.00016$ \\
\hline \hline
\end{tabular}

TABLE III: Absolute dimensionless Helmholtz free energy $\left(\beta f=\beta F / N_{m o l}\right)$ calculation of LiI (per ion pair) at 300 $\mathrm{K}$ for varying system size using MSD-based springs. See supplementary material for details of the column headings shown in this table.

\begin{tabular}{|c|c|c|c|c|c|}
\hline \hline$N_{\text {mol }}$ & $\beta f_{E C}$ & $\beta\left(f_{E C}^{C M}-f_{E C}\right)^{*}$ & $\beta \Delta f_{E C \rightarrow C}^{C M}$ & $\beta\left(f_{C}-f_{C}^{C M}\right)^{*}$ & $\beta f_{C}$ \\
\hline 864 & -3.95800 & -0.01603 & $-304.01438 \pm 0.00016$ & -0.00467 & $-307.99308 \pm 0.00016$ \\
\hline 1372 & -3.95800 & -0.01060 & $-304.01569 \pm 0.00013$ & -0.00294 & $-307.98723 \pm 0.00013$ \\
\hline 2048 & -3.95800 & -0.00739 & $-304.01515 \pm 0.00010$ & -0.00197 & $-307.98251 \pm 0.00010$ \\
\hline 2916 & -3.95800 & -0.00537 & $-304.01679 \pm 0.00009$ & -0.00138 & $-307.98154 \pm 0.00009$ \\
\hline \hline$N_{\text {mol }} \rightarrow \infty$ & -3.95800 & 0.00000 & $-304.01730 \pm 0.00021$ & 0.00000 & $-307.97607 \pm 0.00014$ \\
\hline \hline
\end{tabular}

TABLE IV: Absolute dimensionless Helmholtz free energy $\left(\beta f=\beta F / N_{m o l}\right)$ calculation of $\mathrm{NaCl}$ (per ion pair) at 300 $\mathrm{K}$ for varying system size computed using MSD-based springs. See supplementary material for details of the column headings shown in this table.

\begin{tabular}{|c|c|c|c|c|c|}
\hline \hline$N_{\text {mol }}$ & $\beta f_{E C}$ & $\beta\left(f_{E C}^{C M}-f_{E C}\right)^{*}$ & $\beta \Delta f_{E C \rightarrow C}^{C M}$ & $\beta\left(f_{C}-f_{C}^{C M}\right)^{*}$ & $\beta f_{C}$ \\
\hline 864 & -2.32772 & -0.01734 & $-316.56798 \pm 0.00015$ & -0.00449 & $-318.91753 \pm 0.00015$ \\
\hline 1372 & -2.32772 & -0.01143 & $-316.56964 \pm 0.00012$ & -0.00283 & $-318.91162 \pm 0.00012$ \\
\hline 2048 & -2.32772 & -0.00795 & $-316.57104 \pm 0.00010$ & -0.00189 & $-318.90860 \pm 0.00010$ \\
\hline 2916 & -2.32772 & -0.00576 & $-316.57206 \pm 0.00008$ & -0.00133 & $-318.90687 \pm 0.00008$ \\
\hline$N_{m o l} \rightarrow \infty$ & -2.32772 & 0.00000 & $-316.57449 \pm 0.00013$ & 0.00000 & $-318.90223 \pm 0.00013$ \\
\hline \hline
\end{tabular}

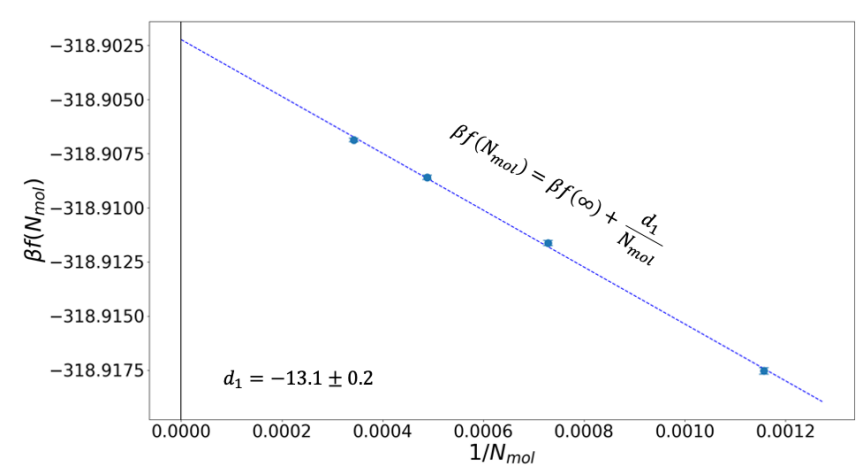

FIG. 2: Intrinsic system-size dependence of $\mathrm{NaCl}$ crystal's free energy

\section{v. CONCLUSIONS}

In this paper we extend the work of Polson et al. ${ }^{15}$ to compute free energies of crystals using an Einstein crystal with fixed center of mass and different spring constants. For calculations that make use of different spring constants, our result provides a revised equation to be used in place of equation (3) in ref. [8]. We also provide necessary conditions to help constrain the center of mass in a molecular dynamics simulations (using MD packages with no built-in capabilities to do the same) employing springs to tether atoms. The free energies of LiI and $\mathrm{NaCl}$ crystals computed using different and equal spring constants are in excellent agreement, thus successfully testing the theory. Our results also demonstrate a reduction in error bars when MSD-based springs are used to optimize the calculations. The significant system size 
dependence of the solid free energy after applying the COM-constraint corrections helps demonstrate the relative magnitudes of the intrinsic and COM-constraint finite-size corrections relative to other contributions to the absolute free energy of the crystals.

\section{Appendix A: Revisiting the Finite Size Correction Derivation $^{15}$}

\section{Computing $Q_{E C}^{C M} / Q_{E C}$ :}

For an $N$ atom Einstein crystal system with uniform spring constants $(\mathrm{k})$ and distinguishable particles,

$$
Q_{E C}=\frac{1}{h^{3 N}} Z_{E C} P_{E C}
$$

where,

$$
\begin{gathered}
Z_{E C}=\int d \mathbf{r}^{N} \prod_{i=1}^{N} \exp \left[-\frac{\beta k}{2}\left\|\mathbf{r}_{i}\right\|^{2}\right]=\prod_{i=1}^{N}\left(\frac{2 \pi}{\beta k}\right)^{3 / 2} \\
P_{E C}=\int d \mathbf{p}^{N} \prod_{i=1}^{N} \exp \left[-\frac{\beta}{2 m_{i}}\left\|\mathbf{p}_{i}\right\|^{2}\right]=\prod_{i=1}^{N}\left(\frac{2 \pi m_{i}}{\beta}\right)^{3 / 2}
\end{gathered}
$$

Similarly,

$$
Q_{E C}^{C M}=\frac{1}{h^{3(N-1)}} Z_{E C}^{C M} P_{E C}^{C M}
$$

Note that in the above equation we have $h^{3(N-1)}$ because with a hard constraint of a fixed center of mass, the system "lives in" a $6 N-6$ dimensional phase space. Equation (A.3) differs from a harmonically restrained system in $6 N$ dimensions, in which case we would still have $h^{3 N}$.

$Z_{E C}^{C M}$ and $P_{E C}^{C M}$ are

$$
\begin{aligned}
Z_{E C}^{C M} & =\int d \mathbf{r}^{N} \prod_{i=1}^{N} \exp \left[-\frac{\beta k}{2} r_{i}^{2}\right] \delta\left(\sum_{i=1}^{N} \mu_{i} \mathbf{r}_{i}\right) \\
& =\left(\frac{\beta k}{2 \pi \sum_{i=1}^{N} \mu_{i}^{2}}\right)^{3 / 2} \prod_{i=1}^{N}\left(\frac{2 \pi}{\beta k}\right)^{3 / 2} \\
& =\left(\frac{\beta k}{2 \pi \sum_{i=1}^{N} \mu_{i}^{2}}\right)^{3 / 2} Z_{E C} \\
P_{E C}^{C M} & =\int d \mathbf{p}^{N} \prod_{i=1}^{N} \exp \left[-\frac{\beta}{2 m_{i}} p_{i}^{2}\right] \delta\left(\sum_{i=1}^{N} \mathbf{p}_{i}\right) \\
& =\left(\frac{\beta}{2 \pi M}\right)^{3 / 2} \prod_{i=1}^{N}\left(\frac{2 \pi m_{i}}{\beta}\right)^{3 / 2} \\
& =\left(\frac{\beta}{2 \pi M}\right)^{3 / 2} P_{E C}
\end{aligned}
$$

where $M=\sum_{i=1}^{N} m_{i}$.

Therefore,

$$
\frac{Z_{E C}^{C M}}{Z_{E C}}=\left(\frac{\beta k}{2 \pi \sum_{i=1}^{N} \mu_{i}^{2}}\right)^{3 / 2}
$$

$$
\frac{P_{E C}^{C M}}{P_{E C}}=\left(\frac{\beta}{2 \pi M}\right)^{3 / 2}
$$

Now, using (A.1) and (A.3),

$$
\begin{aligned}
\frac{Q_{E C}^{C M}}{Q_{E C}} & =h^{3}\left(\frac{Z_{E C}^{C M}}{Z_{E C}}\right)\left(\frac{P_{E C}^{C M}}{P_{E C}}\right) \\
& =\left(\frac{\beta k}{2 \pi \sum_{i=1}^{N} \mu_{i}^{2}}\right)^{3 / 2}\left(\frac{\beta h^{2}}{2 \pi M}\right)^{3 / 2}
\end{aligned}
$$

2. Computing $Q_{C} / Q_{C}^{C M}$ :

$$
Q_{C}=\frac{1}{h^{3 N}} Z_{C} P_{C}
$$

where:

$$
Z_{C}=\int d \mathbf{r}^{N} \prod_{i=1}^{N} \exp \left[-\beta U\left(\mathbf{r}^{N}\right)\right]
$$

$P_{C}=\int d \mathbf{p}^{N} \prod_{i=1}^{N} \exp \left[-\frac{\beta}{2 m_{i}}\left\|\mathbf{p}_{i}\right\|^{2}\right]=\prod_{i=1}^{N}\left(\frac{2 \pi m_{i}}{\beta}\right)^{3 / 2}$

now using equation (13) from Polson et al. ${ }^{15}$,

$$
\begin{aligned}
\frac{Z_{C}}{Z_{C}^{C M}} & =\frac{\int d \mathbf{r}^{N} \prod_{i=1}^{N} \exp \left[-\beta U\left(\mathbf{r}^{N}\right)\right]}{\int d \mathbf{r}^{N} \prod_{i=1}^{N} \exp \left[-\beta U\left(\mathbf{r}^{N}\right)\right] \delta\left(\sum_{i=1}^{N} \mu_{i} \mathbf{r}_{i}\right)} \\
& =\frac{1}{\left\langle\delta\left(\sum_{i=1}^{N} \mu_{i} \mathbf{r}_{i}\right)\right\rangle} \\
& =\frac{1}{\wp\left(\mathbf{r}_{C M}=0\right)} \\
& =\frac{V}{N_{m o l}}
\end{aligned}
$$

Note $^{15}$ that $\wp\left(\mathbf{r}_{C M}\right)$ is the probability distribution function of the center of mass. Since, the probability distribution of the center of mass of the lattice is evenly distributed over a volume equal to that of the Wigner-Seitz cell of the lattice positioned at the center of the volume 
over which we carry out the integration in the partition function. It follows for one molecule per Wigner-Seitz cell: $\wp\left(\mathbf{r}_{C M}=0\right)=N_{\text {mol }} / V$.

Since $P_{C}=P_{E C}$ we have from equation (A.5b)

$$
\frac{P_{C}}{P_{C}^{C M}}=\left(\frac{\beta}{2 \pi M}\right)^{-3 / 2}
$$

Therefore,

$$
\begin{aligned}
\frac{Q_{C}}{Q_{C}^{C M}} & =h^{-3}\left(\frac{Z_{C}}{Z_{C}^{C M}}\right)\left(\frac{P_{C}}{P_{C}^{C M}}\right) \\
& =\left(\frac{V}{N_{\text {mol }}}\right)\left(\frac{\beta h^{2}}{2 \pi M}\right)^{-3 / 2}
\end{aligned}
$$

\section{Free energy of the crystal:}

Using equations (A.6) and (A.11), we have

$$
\begin{aligned}
\beta F_{C} & =\sum_{i=1}^{N} \ln \left(\frac{\beta k \Lambda_{i}^{2}}{2 \pi}\right)^{3 / 2} \\
& -\ln \left[\left(\frac{\beta k}{2 \pi \sum_{i=1}^{N} \mu_{i}^{2}}\right)^{3 / 2}\left(\frac{\beta h^{2}}{2 \pi M}\right)^{3 / 2}\right] \\
+ & \beta \int_{\lambda=0}^{\lambda=1}\left\langle\frac{d U}{d \lambda}\right\rangle_{\lambda} d \lambda \\
- & \ln \left[\left(\frac{V}{N_{m o l}}\right)\left(\frac{\beta h^{2}}{2 \pi M}\right)^{-3 / 2}\right] \\
\beta F_{C} & \left.=\sum_{i=1}^{N} \ln \left(\frac{\beta k \Lambda_{i}^{2}}{2 \pi}\right)^{3 / 2}{ }^{\lambda=1} / \frac{d U}{d \lambda}\right\rangle_{\lambda}^{C M} d \lambda \\
& \left.+\beta \int_{\lambda=0}^{\lambda / 2}\left(\frac{\beta k}{2 \pi \sum_{i=1}^{N} \mu_{i}^{2}}\right)\right] \\
& -\ln \left[\left(\frac{V o l}{N_{m o l}}\right)\right]
\end{aligned}
$$

where, $\Lambda_{i}=\left(\beta h^{2} /\left(2 \pi m_{i}\right)\right)^{1 / 2}$

Note that the log terms in equation (A.12b) have been combined to give a dimensionless argument to the logarithms.

\section{SUPPLEMENTARY MATERIAL}

See supplementary material for details of the column headings shown in Table II, III, and IV, Mathematica result of $Z_{E C, x}^{C M}$, details to ensure zero net external force due to springs, plots of the T.I. integrands, details of the error propagation and autocorrelation time results.

\section{ACKNOWLEDGMENTS}

We are grateful for the financial support provided by Eli Lilly. Use was made of computational facilities purchased with funds from the National Science Foundation (CNS- 1725797) and administered by the Center for Scientific Computing (CSC). The CSC is supported by the California NanoSystems Institute and the Materials Research Science and Engineering Center (MRSEC; NSF DMR 1720256) at UC Santa Barbara.

\section{DATA AVAILABILITY}

The data that supports the findings of this study are available within the article [and its supplementary material]

${ }^{1}$ W. G. Hoover and F. H. Ree, "Use of computer experiments to locate the melting transition and calculate the entropy in the solid phase," J. Chem. Phys. 47, 4873-4878 (1967).

${ }^{2}$ W. G. Hoover and F. H. Ree, "Melting transition and communal entropy for hard spheres," J. Chem. Phys. 49, 3609-3617 (1968).

${ }^{3}$ D. Frenkel and A. J. Ladd, "New monte carlo method to compute the free energy of arbitrary solids. application to the fcc and hcp phases of hard spheres," J. Chem. Phys. 81, 3188-3193 (1984).

${ }^{4}$ C. Vega and E. G. Noya, "Revisiting the frenkel-ladd method to compute the free energy of solids: The Einstein molecule approach," J. Chem. Phys. 127, 154113 (2007).

${ }^{5}$ E. G. Noya, M. Conde, and C. Vega, "Computing the free energy of molecular solids by the Einstein molecule approach: Ices xiii and xiv, hard-dumbbells and a patchy model of proteins," J. Chem. Phys. 129, 104704 (2008).

${ }^{6}$ J. Aragones, C. Valeriani, and C. Vega, "Note: Free energy calculations for atomic solids through the Einstein crystal/molecule methodology using gromacs and lammps," J. Chem. Phys. 137, 146101 (2012).

${ }^{7}$ J. M. Polson and D. Frenkel, "Calculation of solid-fluid phase equilibria for systems of chain molecules," J. Chem. Phys. 109, 318-328 (1998).

${ }^{8}$ J. Anwar, D. Frenkel, and M. G. Noro, "Calculation of the melting point of $\mathrm{NaCl}$ by molecular simulation," J. Chem. Phys. 118, 728-735 (2003).

${ }^{9}$ A. Benavides, J. Aragones, and C. Vega, "Consensus on the solubility of $\mathrm{NaCl}$ in water from computer simulations using the chemical potential route," J. Chem. Phys. 144, 124504 (2016).

${ }^{10} \mathrm{~L}$. Li, T. Totton, and D. Frenkel, "Computational methodology for solubility prediction: Application to the sparingly soluble solutes," J. Chem. Phys. 146, 214110 (2017).

${ }^{11}$ M. A. Bellucci, G. Gobbo, T. K. Wijethunga, G. Ciccotti, and B. L. Trout, "Solubility of paracetamol in ethanol by molecular dynamics using the extended Einstein crystal method and experiments," J. Chem. Phys. 150, 094107 (2019).

${ }^{12}$ J. Anwar, C. Leitold, and B. Peters, "Solid-solid phase equilibria in the NaCl-KCl system," J. Chem. Phys. 152, 144109 (2020).

${ }^{13}$ E. C. Dybeck, N. P. Schieber, and M. R. Shirts, "Effects of a more accurate polarizable hamiltonian on polymorph free energies computed efficiently by reweighting point-charge potentials," J. Chem. Theory Comput. 12, 3491-3505 (2016).

${ }^{14}$ M. Yang, E. Dybeck, G. Sun, C. Peng, B. Samas, V. M. Burger, Q. Zeng, Y. Jin, M. A. Bellucci, Y. Liu, et al., "Prediction of the relative free energies of drug polymorphs above zero kelvin," Cryst. Growth Des. 20, 5211-5224 (2020).

${ }^{15}$ J. M. Polson, E. Trizac, S. Pronk, and D. Frenkel, "Finite-size corrections to the free energies of crystalline solids," J. Chem. Phys. 112, 5339-5342 (2000).

${ }^{16}$ D. Frenkel and B. Smit, Understanding molecular simulation: from algorithms to applications, Vol. 1 (Elsevier, 2001). 
${ }^{17}$ J. Aragones, E. G. Noya, C. Valeriani, and C. Vega, "Free energy calculations for molecular solids using gromacs," J. Chem. Phys. 139, 034104 (2013).

${ }^{18}$ R. K. Reddy A and S. N. Punnathanam, "Calculation of excess free energy of molecular solids comprised of flexible molecules using einstein molecule method," Molecular Simulation 44, 781788 (2018).

${ }^{19}$ S. Plimpton, "Fast parallel algorithms for short-range molecular dynamics," J. Comput. Phys. 117, 1-19 (1995).

${ }^{20}$ R. Freitas, M. Asta, and M. De Koning, "Nonequilibrium freeenergy calculation of solids using LAMMPS," Computational Materials Science 112, 333-341 (2016).

${ }^{21}$ E. A. Mastny and J. J. de Pablo, "Direct calculation of solidliquid equilibria from density-of-states monte carlo simulations,"
J. Chem. Phys. 122, 124109 (2005).

${ }^{22}$ J. G. Kirkwood, "Statistical mechanics of fluid mixtures," J. Chem. Phys. 3, 300-313 (1935).

${ }^{23}$ M. Mezei and D. Beveridge, "Free energy simulations," Ann. New York Acad. Sci. 482, 1-23 (1986).

${ }^{24}$ I. S. Joung and T. E. Cheatham III, "Determination of alkali and halide monovalent ion parameters for use in explicitly solvated biomolecular simulations," J. Phys. Chem. B 112, 90209041 (2008)

${ }^{25}$ P. P. Ewald, Ann. Phys. 369, 253-287 (1921).

${ }^{26} \mathrm{R}$. W. Hockney and J. W. Eastwood, Computer simulation using particles (Adam Hilger, NY, 1989).

${ }^{27}$ M. Abramowitz and I. A. Stegun, Handbook of mathematical functions: with formulas, graphs, and mathematical tables, Vol. 55 (Courier Corporation, 1965). 